\title{
Waste Water Treatment by Environmental Microbiology
}

\author{
Hiral Borasiya and *Maulin P Shah \\ Industrial Waste Water Research Lab, Division of Applied \& Environmental Microbiology, India
}

Submission: February 23, 2017; Published: March 10, 2017

*Corresponding author: Maulin P Shah, Industrial Waste Water Research Lab, Division of Applied \& Environmental Microbiology, Enviro Technology Limited, India, Email: shahmp@beil.co.in

\section{Mini Review}

Even formerly the discovery of the life of microorganisms, the man produced fermented foods and feed. Also degrading activity of microorganisms used in human waste, which is naturally biodegradable. However, for large-scale treatment, the man has had to devise artificial processes competition degrading activity of naturally occurring microorganisms. Microbiologists, engineers and chemists have worked hand in hand in this regard, now we know that for biodegradable materials, other than human waste, the process is also true. These biological systems for waste belonging to one of the largest area for biotechnological applications. Usually the goal of biotechnology products synthesized using an especially pure isolated strain of microorganisms under optimum conditions. Defined nutrient media (source Carbon, energy source, nitrogen, sulphur, phosphorus, trace elements and vitamins) exposed uses. Growth and yield of biomass and cultural conditions Related to the properties of pure strains. The production of antibiotics, a valuable product is an example of this development in the field of traditional biotechnology.

Waste treatment Biotechnology is significantly different from traditional processes. First, there are no profit-oriented commercial products are produced apart from cleaner wastewater for a better environment that will soon be invaluable. Other wastes are often mixed. Finally, a pure culture cannot be used and cannot rely on the natural mixed population or the enrichment of mixed cultures, if possible. The magnitude of the economy is therefore very different. Industrial microbiologists recognized the utility of microorganisms in waste treatment in 1914, with the development of a highly versatile biological treatment unit known as activated sludge processes. This process depends on a mixed culture of natural microorganisms, each having the capacity to degrade the components of a fraction of waste and the possibilities coexisting together. Recent advances in research and development to improve Flock biological training and reduce inoculation of sludge above the system treat specially adapted and cultured microorganisms. Currently, the crop that is capable of degrading certain priority toxic substances and undesirable compounds in the waste streams are already available. With the advent of genetic engineering, some microorganisms can be designed and adapted to degrade certain types of waste. However, the release of these genetically modified micro-organisms can negatively affect the ecosystem as the chemical waste nightmare that man has created. Upon learning this, man has now developed an in-depth research into the sequelae of these strains after its application on a large scale.

The present state of the art is complemented by the existing bacterial population of bacterial strains which are capable of reducing the higher level or with strains which are capable of decomposing compounds of those previously considered non-biodegradable. This paper discusses the poor degradable substances found in industrial wastes that are now subject to microbial decomposition. Aerobic metabolism consists of two processes: first, the transfer of electrons from the substrate to organic oxygen - as a source of energy for the cells, and secondly, by adding oxygen organic substrate - preparing the substrate for metabolism. The degradation of xenobiotics and difficult degradable compounds is important for the second part. The aromatic compounds, the secession of the cycles depends on the oxygen. The availability of molecular oxygen for the reaction depends on several enzymes. Studies on the anaerobic degradation of organic compounds are limited, but the process will be important and attractive in the near future. In nature, many microorganisms used for the growth of hydrocarbons and the energy source. The microbes oxidize the terminal methyl group in the aliphatic hydrocarbon. Become a hydrocarbon fatty acid. Generally, each species can degrade limited types of hydrocarbons. For example, Methanomonas methanooxidans can attack only methane, while Nocardia Paraffinicum and certain species of Pseudomonas can use several hydrocarbons, not all of which are necessarily present in the oil.

Benzenoid structures are the most common organic compounds in nature, and microorganisms attack them fairly 
well. However, aromatic polycyclics and those with rare substituents (e.g., polychlorinated biphenyls) are difficult to decompose. The degradation of the aromatic compounds begins to split the ring. Examples of microbes that carry this attack include Pseudomonas stutzeri, Pseudomonas mendocina, Psudomonas putida and P. ovals.

Halogen compounds are used as solvents, aerosols, lead scavengers, fumigants and nematicides, among others. The species of Pseudomonas and Xantobacter autophicus are capable of degrading these compounds. The halogenated aromatic compounds are used as solvents, lubricants, intermediates in the synthesis, insulators, plasticizers, etc., degrade with respect to the halocatocalic formation or before the cleavage of the dehalogenation of the ring. Examples of such microorganisms are Pseudomonas and Athrobacter species. Nitro aromatic compounds used in the production of paints, medicines, pesticides, explosives and industrial solvents are toxic. The simpler compounds are completely biodegradable nitro aromatic complexes such as 2,4,6-trinitrotoluene is not degraded. Under aerobic conditions, the polymerization may take place under anaerobic conditions; Amine conversion can take place. Polychlorinated biphenyls used in transformer oil, capacitor dielectrics and heat transfer fluids are toxic to animals and humans. The species Acinetobacter and Alcaligenes are able to transform many PCBs. PCBs containing more than four are resistant to chlorine degradation.

Xenobiotics and pollutants are released into the environment by point sources or dispersed by consumers and users of the final product. With appropriate destruction information regarding the pollution of this waste consumed and with the appropriate control of the system institution efficient for the collection and treatment of waste, often complex physical and chemical properties of fractions of waste can be controlled. Thus, the use of these microorganisms in the degradation of the contaminants can be optimized. Experiments with pure cultures of the individual substrates form the basis of the collective knowledge of the biosynthetic pathways of the compounds which are not very degradable in microorganisms. After pure culture is available, the development of biotechnological processes is possible. For the isolation of microorganisms with biodegradable resources, microbiologists use the technique of enrichment culture. The procedure is to allow microorganisms with growth potential in a medium with low degradable compounds and a nutrient source limiting essential growth. Only micro-organisms that can decompose this substance will grow.

A number of subcultures evaluate the success of the enrichment. Wastewater, where many microorganisms come in contact with xenobiotics, is a common source of enrichment for bacteria with degradation capacity. However, isolates from the natural environment in which compounds of interest have been found are generally successful. These include samples from industrial production lines, soil treated with pesticides, landfills and wastewater treatment. Fermentation processes are generally conventional, from the preparation of capillary inoculums and sequential seeding to fermentators up to 10,000 litres. Although high, sterile mixed conditions are maintained to protect against contamination by salmonella, staphylococci and streptococci. Cultural conditions are maintained products of microorganisms that are repressed and conditioned their final environment. Methods of centrifugation or filtration which are used for cell concentration. Spore formation was air dried and not lyophilized. The cultures are then mixed with additives before final packaging. For significant progress in the microbial digestion of waste, it is necessary to identify organic chemicals that resist degradation in conventional waste treatment plants. After identification, the dismantling of existing plant protection products can now be improved or specialized technology suitable for their biodegradation can be obtained.

Among the organic chemicals in the EPA list of priority contaminants are pesticides and metabolites, phenolic compounds, halogenated, aroma nitro aromatics, chloroaromatics, PCBs, phthalate esters, polycyclic aromatic hydrocarbons and nitrosamines. This EPA list is helpful in determining the direction of research to improve the system for waste water treatment. The biomass consists of cellulose, hemicellulose and lignin. Lignin acts as a cementing material in the lignocellulosic material, and protects the structure of microbial degradation. Biodegradation of lignin is important because of the increasing number of industrial uses. Lignosulfonate, which are resistant to biodegradation of lignin, waste sulphite process products in the pulp and paper. White rot fungi can degrade lignosulfonates. Other mushrooms and mixed microbes promote precipitation through poliplerization. Commercial detergents containing $10 \%$ to $20 \%$ of surface-active agents for cleaning. Anionic surfactants are not biodegradable. Substituted level alkilbcnzcnsulfonata is more biodegradable, but are deposited in the sewer system. Cultures of microorganisms adapted for degradation of alkyl sulphates and alkyl sulfonates was very fast. Currently, 3,500 buoys are in use (40,000 colours and pigments to 7,000 different chemical structure). Therefore, we cannot generalize their biodegradability. Textiles and paint industries are responsible for replacing colours in the environment, although in small quantities. The model for the biodegradation activity Pteudomon $\mathrm{M}$ type on azo dyes for the production of biomass, $\mathrm{CO}_{2}, \mathrm{H}_{2} \mathrm{O}$ and $\mathrm{NH}_{3}$. In real situations, using a mixed culture adapted microorganisms.

Research on biodegradation of xenobiotics and pollutants to accumulate. The results of microbial, biological and genetic research will ultimately improve the practical application of methods of treatment on an industrial scale. With a higher level of sophistication to be achieved, more and more specific microbial species for biodegradation of the waste stream will be used. Ideally, the waste using microorganisms should emulate industrial fermenters. However, aseptic conditions are not possible, and the system is faced with a different 
composition, temperature and volume. With the ever changing toxic loads, micro-organisms can be harmed. Regularly feeding can wash desirable strains. However, regardless of the problems encountered, several microbial processes have now been successful. Special mixed culture of bacteria mutants for specific types of waste are now available in the market. They are far more efficient in how they consume less energy than conventional systems. Biological processes are now more attractive, effective and most importantly, economical.

\section{Conclusion}

With genetic engineering technology, some of the activities of microbial cultures are now a reality. This has led to new approaches to the treatment of waste. New microbial species that are genetically modified, and that cannot be found in nature may be patentable. The accumulation of research and study, as well as the results of the current state of the art application of biotechnology is the treatment of waste will lead to a more efficient system.

\section{Your next submission with Juniper Publishers will reach you the below assets}

- Quality Editorial service

- Swift Peer Review

- Reprints availability

- E-prints Service

- Manuscript Podcast for convenient understanding

- Global attainment for your research

- Manuscript accessibility in different formats

( Pdf, E-pub, Full Text, Audio)

- Unceasing customer service

Track the below URL for one-step submission https://juniperpublishers.com/online-submission.php 